\title{
Observations of geomagnetically induced currents in the Australian power network
}

\author{
R. A. Marshall, ${ }^{1}$ H. Gorniak, ${ }^{2}$ T. Van Der Walt, ${ }^{2}$ C. L. Waters, ${ }^{3}$ M. D. Sciffer, ${ }^{3}$ M. Miller, ${ }^{2}$ \\ M. Dalzell, ${ }^{4}$ T. Daly, ${ }^{5}$ G. Pouferis, ${ }^{5}$ G. Hesse, ${ }^{6}$ and P. Wilkinson ${ }^{1}$
}

Received 14 August 2012; revised 19 October 2012; accepted 3 November 2012; published 27 January 2013.

[1] Infrastructures such as pipelines and power networks at low-middle latitude regions have historically been considered relatively immune to geomagnetically induced currents (GICs). Over the past decade there have been an increasing number of investigations into the impact of GICs in long grounded conductors at these latitudes. The Australian region power network spans thousands of kilometers from low to middle latitudes. The approaching maximum of solar cycle 24 and recent findings of studies into power networks located at similar latitudes have stimulated the Australian power industry to better understand this phenomenon in their region. As a result, a pilot study to compare space weather activity with in situ GIC monitors at strategic locations within the power network was initiated. This paper provides some results from the first of these operational GIC monitors during a modest geomagnetic storm, showing the first observational evidence of space weather well correlated with GICs measured in the Australian power network. Transformer neutral currents show a high degree of similarity with the geoelectric field derived from the closest available geomagnetic observatory.

Current maxima of 4-5 amperes were observed in association with geoelectric field values of $\sim 0.06-0.07$ volts per kilometer. This paper also discusses the GIC measurements obtained during this storm in terms of the space weather drivers and the considerably larger geoelectric field values anticipated during larger geomagnetic storms.

Citation: Marshall, R. A., et al. (2013), Observations of geomagnetically induced currents in the Australian power network, Space Weather, 11, 6-16, doi:10.1029/2012SW000849.

\section{Introduction}

[2] Geomagnetically induced currents (GICs) flowing in long grounded conductors such as power networks and pipelines are an important element of space weather. Historically, much of the focus has been at high latitudes [Elovaara et al., 1992; Boteler et al., 1998; Viljanen et al., 1999; Bolduc, 2002; Lam et al., 2002; Béland and Small, 2004; Kappenman, 2005; Pirjola, 2005; Pulkkinen et al., 2003, 2005; Wik et al., 2008]. Recent studies have also considered these infrastructures at middle-low latitudes

\footnotetext{
${ }^{1}$ IPS Radio and Space Services, Bureau of Meteorology, Sydney, New South Wales, Australia.

${ }^{2}$ Australian Energy Market Operator Limited, Melbourne, Australia.

${ }^{3}$ School of Mathematical and Physical Sciences, University of Newcastle, Australia.

${ }^{4}$ Transpower New Zealand Limited, Wellington, New Zealand.

${ }^{5}$ ElectraNet Pty Limited, Rymill Park, Adelaide.

${ }^{6}$ Powerlink Queensland, Virginia, Queensland.

Corresponding author: R. A. Marshall, IPS Radio and Space Services, Bureau of Meteorology, Sydney, NSW 2000, Australia. (r.marshall@ips.gov.au)
}

[Kappenman, 2003; Gaunt and Coetzee, 2007; Trivedi et al., 2007; Liu et al., 2009a, 2009b; Ngwira et al., 2008, 2009; Watari et al., 2009; Marshall et al., 2011, 2012; Torta et al., 2012]. GICs have been observed in the Australian region pipeline network [Martin, 1993; Trichtchenko, 2005; Waters et al., 2006; Marshall et al., 2010], but as yet have not been observed in the Australian region power network in association with space weather activity. Zanussi [1993] made in situ measurements on the transformer neutral line at the end of a 132 kilovolt transmission line in northern Queensland (QLD) over a period of 5 months and compared these measurements with magnetometer data from a nearby proton precession magnetometer. The author concluded that no GICs were observed within experimental limits and was unsuccessful in showing an association between in situ GIC measurements and the geomagnetic field recordings. The null result was attributed to possible effects of soil resistivity and distance from the auroral electrojet region and the author suggested a longer data campaign should be conducted. This paper provides some of the first in situ observations of GICs in the Australian power network associated with variations of the geomagnetic field during modest geomagnetic activity. 


\section{MARSHALL ET AL.: GICS IN AUSTRALIAN POWER NETWORK}

[3] The Australian region power network (Figure 1a) spans thousands of kilometers from low to middle latitudes with interconnects between state grids. The Australian Energy Market Operator (AEMO) operates the National Energy Market in eastern Australia, working closely with the Transmission Network Service Provider's (TNSPs) in each state. Recent studies of GICs in middle-low latitude regions such as South Africa [Gaunt and Coetzee, 2007] and New Zealand [Dalzell, 2011; Marshall et al., 2012] have stimulated increased interest in the possible impacts of space weather to the Australian region power grid. AEMO convenes regular meetings of the Power System Security Working Group, which has recently focused on this issue with input from various groups such as the Australian Bureau of Meteorology's Space Weather branch (BOM/ IPS), Transpower Ltd New Zealand, Eskom South Africa, the TNSPs, AEMO representatives, and Australian Universities. One of the outcomes of these meetings was an initiative to install GIC monitoring devices at a small number of strategically selected locations across the Australian power network as part of a collaborative "pilot study" between AEMO, the TNSPs, the BOM, and a number of Australian Universities [Gorniak, 2011]. It was envisaged that the monitors would be operational from the middle of 2012 . This paper presents data recorded by the first two of these in situ monitors correlated with space weather activity as part of this initiative. This paper also discusses the implications of these results in terms of more significant geomagnetic activity.

\section{Data Acquisition and Analysis}

[4] Comprehensive modeling of GICs in power networks requires knowledge of many factors such as magnetospheric and ionospheric source current geometry [Pirjola, 1982, 2002; Pirjola and Viljanen, 1998; Viljanen et al., 1999; Pulkkinen et al., 2003], Earth conductivity models [Viljanen and Pirjola, 1994; Kappenman, 2003; Thomson et al., 2005; Ngwira et al., 2008, 2009], network orientation, transmission line length, and line/transformer/Earth resistances [Lehtinen and Pirjola, 1985; Viljanen and Pirjola, 1994; Pirjola, 2000; Pulkkinen et al., 2012]. Such modeling for the Australian region network would provide locations most likely to observe GICs. In the absence of such an analysis, the strategic locations for installation of the GIC monitors were selected using a combination of the following "rules-of-thumb":

1. GICs are typically larger in higher power transmission lines and transformers [Kappenman, 2004].

2. Geomagnetic field variations at high latitudes are generally larger in the geomagnetic north-south direction [Beamish et al., 2002] resulting in geoelectric fields that drive GICs generally being larger in the geomagnetic east-west direction [Elovaara et al., 1992]. To a first approximation this is also assumed for the Australian region.

3. GICs are typically expected to be larger in southern Australian regions [Marshall et al., 2011] although more northern regions (low-middle latitudes) should not be excluded due to geomagnetic sudden impulse events [Marshall et al., 2012].

[5] Considering the above, in conjunction with the practical considerations of installation, the following locations were selected for in situ GIC monitoring devices:

Tasmania - Chapel St and George Town (220 kilovolts) Victoria - Hazelwood Terminal Station and Moorabool Terminal Station (500 kilovolts)

South Australia (SA) - Para (275 kilovolts)

New South Wales - Bannaby and Bayswater (500 kilovolts) Queensland - Middle Ridge (330/275 kilovolts)

[6] These locations can be found in Figure 1a and the simplified map of Figure $1 \mathrm{~b}$.

[7] The GIC monitoring devices are Hall effect current transducers (LEM model LT 505-S), which were installed onto the transformer neutral line connection to earth. The data from these monitors are configured into a supervisory control and data acquisition (SCADA) system and transferred to both the respective TNSP control center and onto AEMO. This study compares data from in situ monitors at the Para (SA) and Middle Ridge (QLD) sites with geoelectric field data derived from the nearest geomagnetic observatory. The corresponding geomagnetic observatory data are obtained from Geoscience Australia's Canberra observatory and the BOM's space weather monitoring site at Culgoora, Narrabri, respectively (Figures $1 \mathrm{a}$ and $1 \mathrm{~b}$ ). Middle Ridge is approximately 400 kilometers north-east of Narrabri and Para is more than 1000 kilometers west of Canberra, having similar latitude. The sample period of the data presented here is 1 minute. The SCADA system at Para was initially configured to measure only positive currents during its early operation including the geomagnetic storm interval studied in this paper. During negative currents the SCADA system recorded zero values. The SCADA system at this site has since been reconfigured to measure currents of both polarities.

[8] Previous studies have derived the geoelectric field from geomagnetic field data in both the time domain [Pirjola, 2002; Hejda and Bochnicek, 2005; Ngwira et al., 2009; Torta et al., 2012] and the frequency domain [Pirjola, 1982, 2002; Trichtchenko and Boteler, 2004]. In the frequency domain, the simplest model assumes the Earth is a uniformly conducting half-space and the magnetic source field can be represented by a vertically downward propagating plane wave. This approach is used in this study and gives the equation of magnetotellurics [e.g., Cagniard, 1953; Pirjola, 1982], which relates the orthogonal electric and magnetic field components at the Earth's surface by the equation:

$$
E_{y}=-\sqrt{\frac{\omega}{\mu_{0} \sigma}} B_{x} e^{i \frac{\pi}{4}}
$$




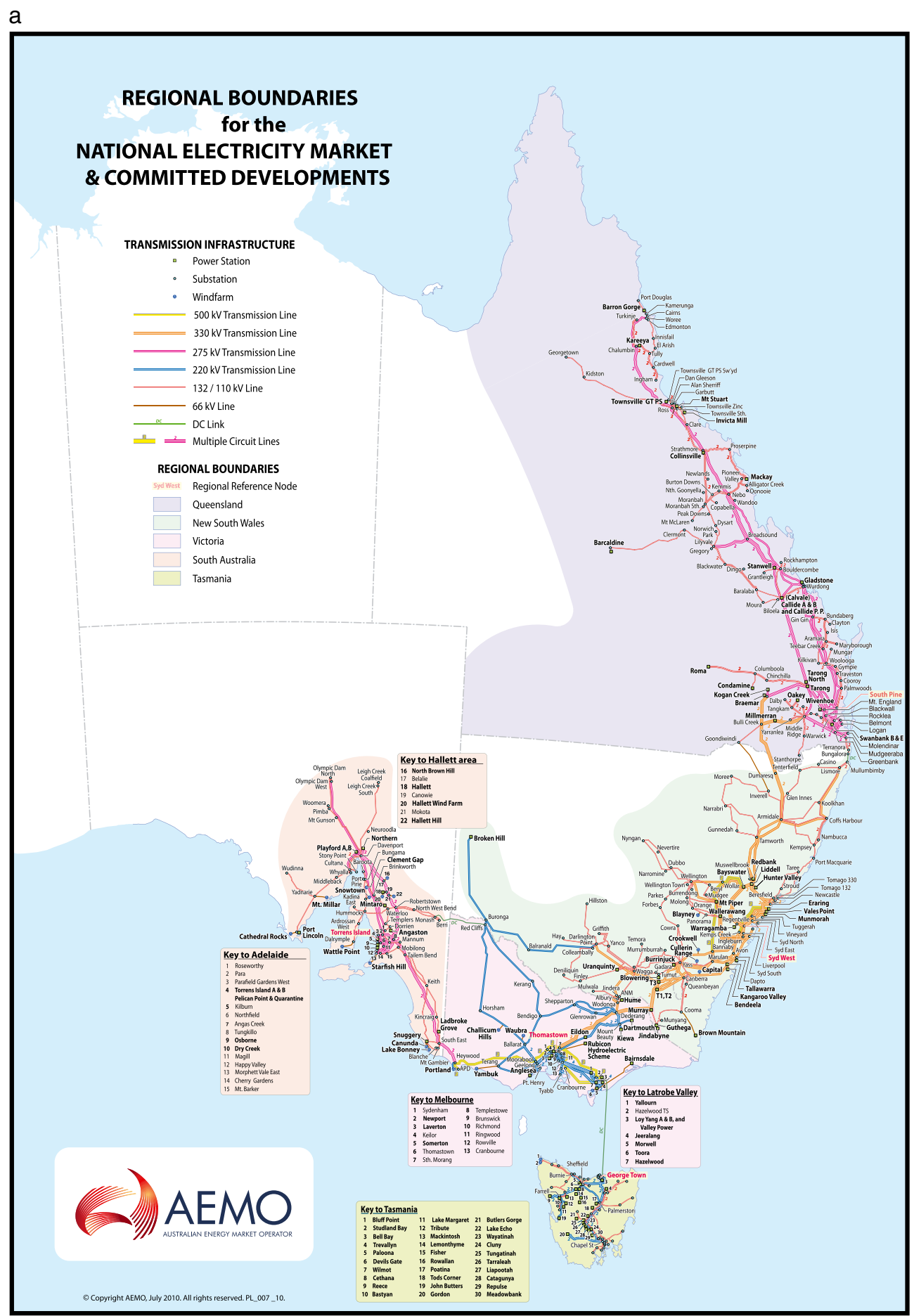

Figure 1. (a) Map showing the power network of eastern Australia. The network extends from the state of Queensland in the north to Tasmania in the south. The map is supplied courtesy of the Australian Energy Market Operator Limited (www.aemo.com.au). All copyright remains with Australian Energy Market Operator Limited. (b) Simplified map showing the locations of in situ monitors in the power network of Figure 1a indicated by the square symbols and the locations of the geomagnetic observatories indicated by the triangle symbols. 


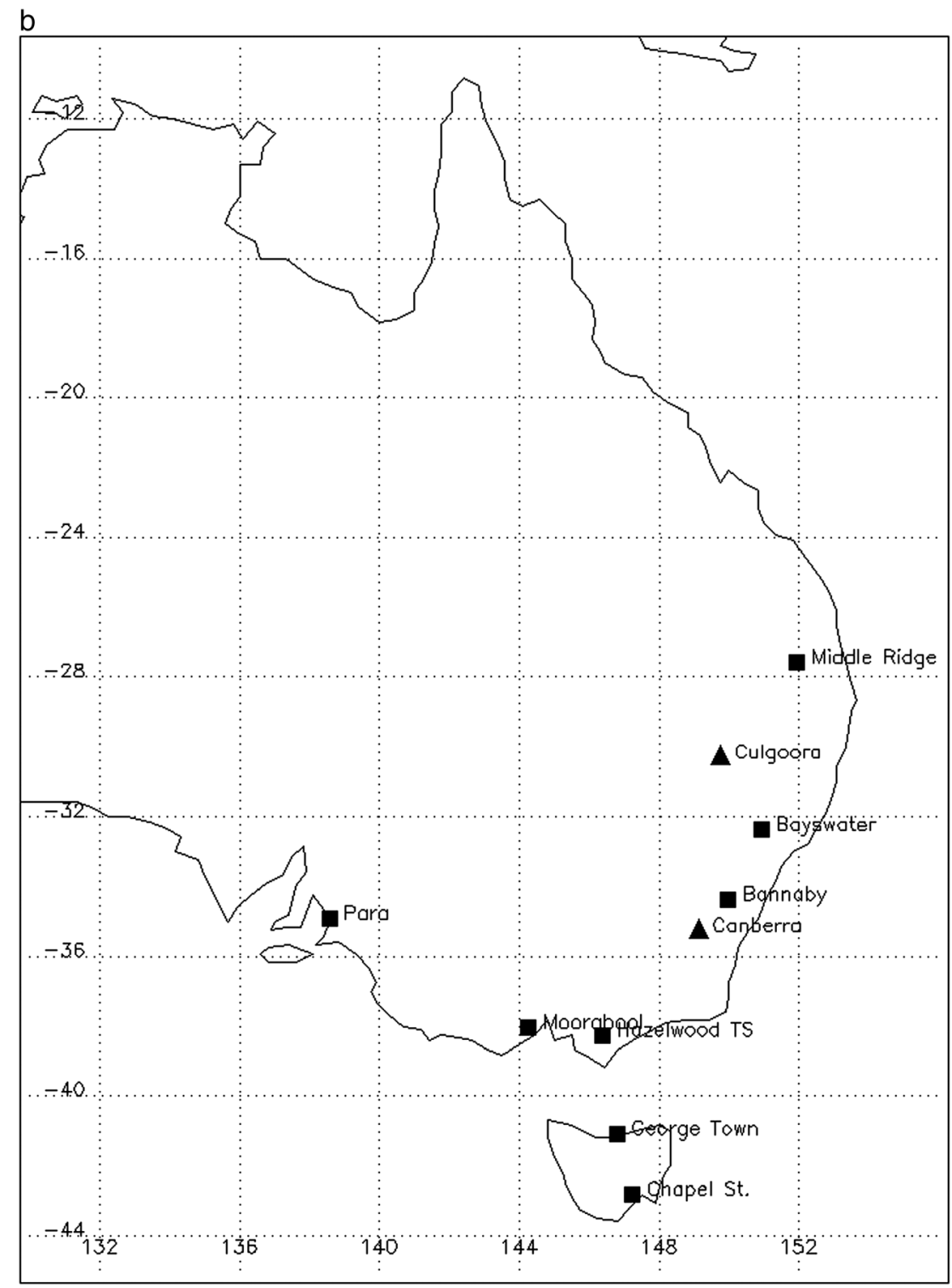

Figure 1. (continued)

[9] Conductivity studies within the Australian region have used, or determined, values for $\sigma$ typically between 0.001 siemens per meter and 0.01 siemens per meter [Campbell et al., 1998; Wang and Chamalaun, 1995; Wang and Lilley, 1999] for signals with skin depths of tens to several hundred kilometers, typical of the signals driving GICs. This study uses a value for $\sigma$ of 0.002 siemens per meter in equation (1) for calculating the geoelectric field. The geoelectric field time series, $E_{y}(t)$, is obtained by applying the reverse Fourier transform to $E_{y}(\omega)$ determined from equation (1). $E_{x}$ may be determined using the analogous expression for $B_{y}$.

[10] The GIC amplitude may be determined for a given transformer/transmission line using

$$
\operatorname{GIC}(t)=a E_{x}(t)+b E_{y}(t),
$$

where the coefficients $a$ and $b$ depend on system parameters and may be determined by inverting GIC measurements and geomagnetic observations [Ngwira et al., 2009; Wik et al., 2008; Pulkkinen et al., 2012].

[11] This study also utilizes GIC indices defined by Marshall et al. [2010, 2011], which give a proxy for the geoelectric field and provide an indicator of GIC-related space weather conditions analogous to $\mathrm{d} B / \mathrm{d} t$ data. GIC indices may be obtained from single component geomagnetic field data processed through a simple frequency 


\section{MARSHALL ET AL:: GICS IN AUSTRALIAN POWER NETWORK}

domain filter, $Z(f)$, with normalized amplitude and phase characteristics represented by

$$
Z(f)=\sqrt{\frac{f}{f_{N}}} e^{i \frac{\pi}{4}}
$$

where $f_{\mathrm{N}}$ is the Nyquist frequency.

[12] The data interval selected for this study was 14-15 July 2012 (UT) during which a modest geomagnetic storm occurred. The planetary $A$-index, $A p$, for these days were 18 and 86 with the minimum $D s t$-index (real time) reaching -125 nanoteslas at 18 UT on 15 July. Comparison of these geomagnetic activity indicators with those of the "2003 Halloween storm" that occurred during solar cycle 23 (for 29-31 October 2003 Ap-indices were 204, 191, and 116, respectively, with Dst-index minima of -383 nanoteslas), indicates that the geomagnetic storm of 14-15 July 2012 was relatively modest. This geomagnetic activity was the result of the Earth being impacted by a coronal mass ejection (CME) originating from NOAA solar region 11520. An X1 flare was observed at 1649 UT on 12 July. At this time the active region longitude was $9^{\circ}$ west of the Sun's central meridian, well located for the associated CME activity to be "geoeffective" although the latitude of the active region $\left(16^{\circ} \mathrm{S}\right)$ most likely reduced the potential impact of the CME somewhat.

\section{Results}

[13] The CME was observed by the ACE satellite at approximately 1725 UT on the 14 July as indicated by the increase in solar wind, $V_{\mathrm{sw}}$, from 390 kilometers per second to 640 kilometers per second at 1738 UT (Figure 2a). In addition to the step increase in solar wind speed, step changes were also observed in the ion temperature, $T$, and total interplanetary magnetic field (IMF) strength, $B_{\mathrm{t}}$, shown in Figure 2a. The increase in density, $N_{\mathrm{p}}$, shown in Figure 2a was relatively small. The increase in dynamic pressure at the Earth's magnetosphere resulted in a small Sudden Impulse (SI) event at 1810 UT on 14 July of 29 nanoteslas as determined from the BOM's space weather monitoring network of magnetometers. Examples of the SI step increase in the geomagnetic field $x$-component and the associated spike in $\mathrm{GIC}_{y}$ indices are shown in Figure 3a for Canberra and Culgoora data. Figures $3 \mathrm{a}$ and $3 \mathrm{~b}$ show that the largest $\mathrm{GIC}_{y}$ index observed during the period 14-15 July was associated with this SI event. GIC indices became relatively elevated again during 15 July (Figure $3 b$ ) in association with a period of sustained southward IMF for many hours from approximately 06UT on 15 July as shown in Figure $2 b$.

[14] Figures $4 \mathrm{a}$ and $4 \mathrm{~b}$ show the transformer neutral current measured at Middle Ridge plotted with the geoelectric field $y$-component, $E_{y}$, derived from geomagnetic field $x$-component data recorded at Culgoora for 14 and 15 July (UT), respectively. The largest amplitude of the measured neutral current is approximately 5.3 amperes, coincident with the enhanced GIC index due to the SI event shown in Figure 3a at 1810 UT. Further elevated transformer neutral currents were observed during the period of extended southward IMF (Figure $2 \mathrm{~b}$ ). The similarities between the geoelectric field $E_{y}$ component and the transformer neutral current are obvious and suggest a strong link between these two parameters. The correlation coefficients between the two time series of Figures $4 \mathrm{a}$ and $4 \mathrm{~b}$ are 0.84 and 0.93 , respectively. The high degree of correlation implies $b E_{y} \gg a E_{x}$ and equation (2) may be approximated to GIC $\approx b E_{y}$ for this situation.

[15] Figures $5 a$ and $5 b$ show analogous plots to Figures $4 a$ and $4 \mathrm{~b}$, respectively, for the GIC monitor at Para plotted with the geoelectric field $y$-component, $E_{y}$, derived from geomagnetic field $x$-component data recorded at Canberra. The largest amplitude of the measured transformer neutral current during 14-15 July is approximately 4.1 amperes, again coincident with the SI event of Figure 3a. The similarities between the transformer neutral positive current and the derived geoelectric field are obvious, even when the magnetometer station is some considerable distance away in longitude (Canberra data was adjusted for the local time difference). The correlation coefficients between the two time series of Figures $5 a$ and $5 b$ are 0.40 and 0.59 . The zero values recorded during negative currents, as mentioned previously, have likely resulted in lower correlation coefficients than would otherwise have been observed. This is demonstrated in Figure 6, which shows an analogous plot to Figure 5 for 30 July, when the SCADA system had been configured to measure currents of both polarity. Even though the geomagnetic activity on this day was relatively quiet with an $A p$ index of 15 , the correlation coefficient was 0.65 . In addition, the assumption of a uniform geoelectric field applied over the relatively larger separation distance between Para and Canberra is also likely to have contributed to relatively lower correlation coefficients. Furthermore, appropriate combination of $E_{y}$ and $E_{x}$ in equation (2) would most likely improve correlation values; however, the strong similarities between the time series of Figures 5 and 6 imply $b E_{y}>a E_{x}$ and equation (2) may be roughly approximated to GIC $\sim b E_{y}$ for this situation. The correlation values obtained using $E_{y}$ only are therefore considered sufficient to illustrate the points presented in this paper.

\section{Discussion}

[16] The above results show GICs correlated with space weather activity from the first two of a number of in situ GIC monitors being installed into the Australian power network as part of an initiative to better understand the phenomena in the region. These results provide the first observational evidence of space weather well correlated with GICs in this network. Although the results are only for two locations within a large network, the high degree of similarity with the geoelectric field and the large separation between monitoring sites would suggest that GICs 
(a)
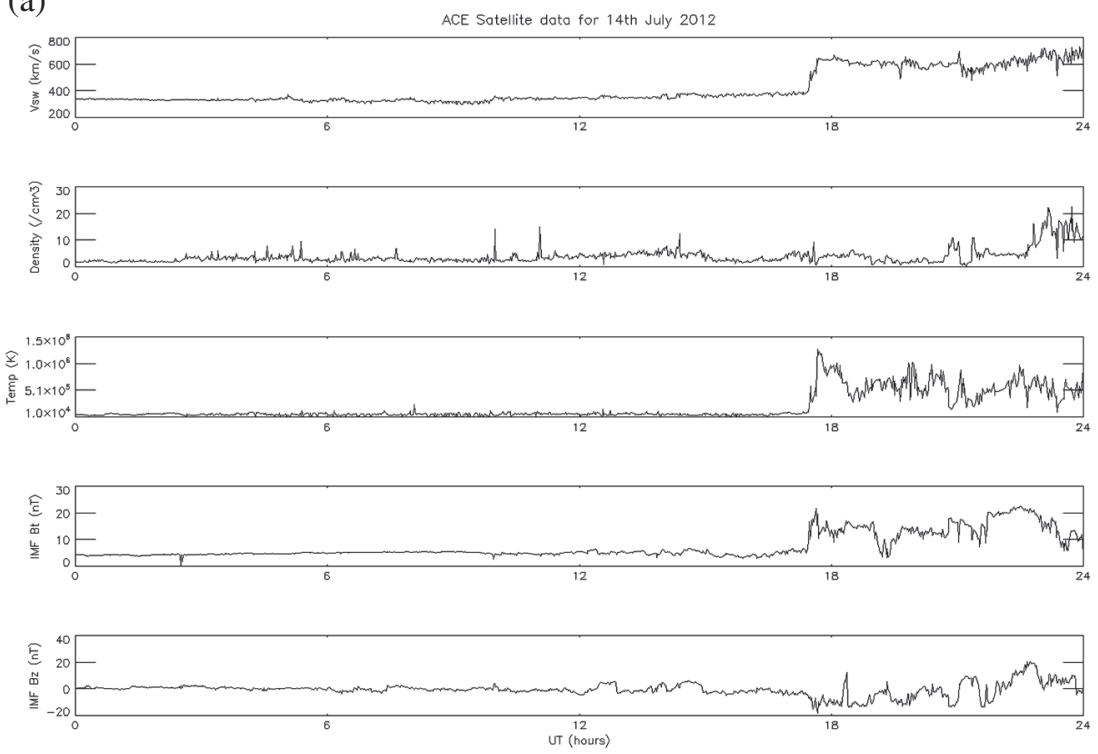

(b)
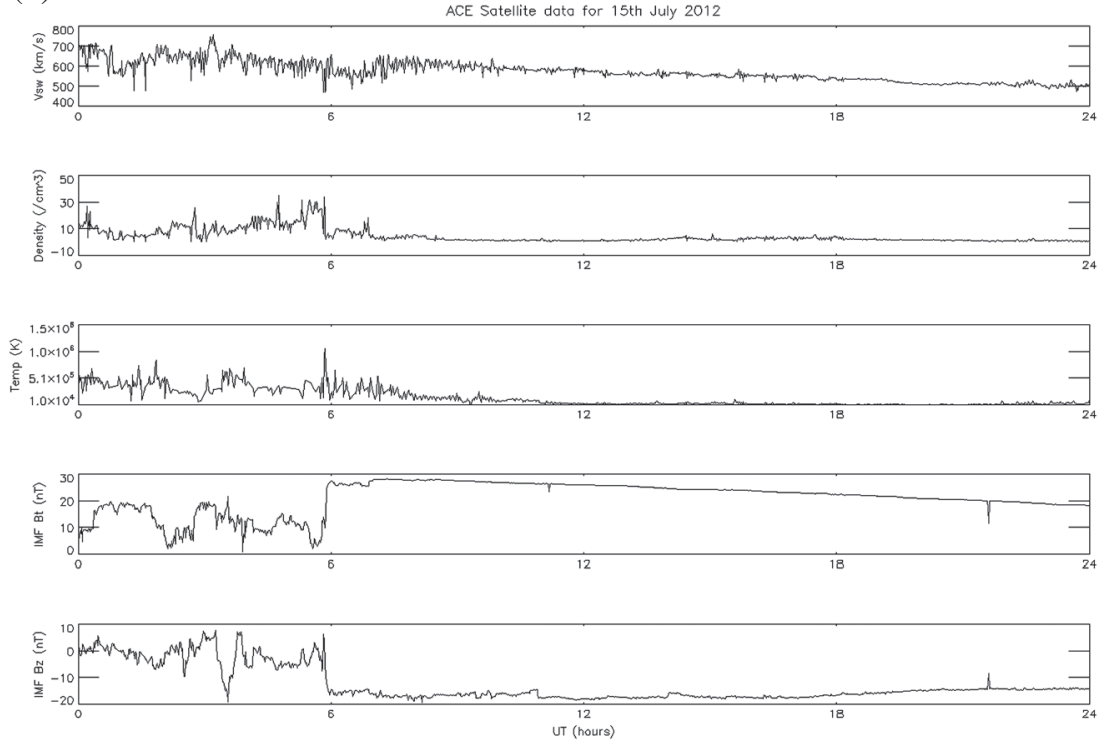

Figure 2. ACE satellite solar wind parameters for (a) 14 July and (b) 15 July 2012 (UT). The five solar wind parameters plotted for both Figures $2 a$ and $2 b$ are from top to bottom: bulk solar wind speed, $V_{\text {swi }}$; density, $N_{\text {p }}$ ion temperature, $T$; IMF total field strength, $B_{\mathrm{t}}$; and IMF $z$-component, $B_{z}$.

are likely to be observed in other parts of the Australian network. Appropriate modeling such as that demonstrated by Viljanen and Pirjola [1994], Thomson et al. [2005], Ngwira et al. [2008, 2009], and Pulkkinen et al. [2012], calibrated with in situ observations, would reveal if relatively larger currents are likely in other parts of the network.

[17] GIC indices are a proxy for the geoelectric field (equation (1) evaluated for a uniform unity conductivity model, also see Marshall et al. [2010, 2011, 2012]), which incorporates the frequency dependence of the magnetic variations (equation (3)). Geoelectric fields have previously been scaled between geomagnetic storms. For example, Pulkkinen et al. [2012] scaled geoelectric field values to provide an estimate of a one-in-hundred-year geomagnetic storm scenario. As such, both GIC indices and geoelectric fields may be scaled to estimate current magnitudes likely to be observed at the two monitoring sites during larger geomagnetic storms. The largest amplitude current observed during the interval 14-15 July (UT) was approximately $4-5$ Amps at both monitoring sites. This was in association with an SI event with a corresponding $\mathrm{GIC}_{y}$ index of approximately 15 and geoelectric 
MARSHALL ET AL.: GICS IN AUSTRALIAN POWER NETWORK

(a)


(b)
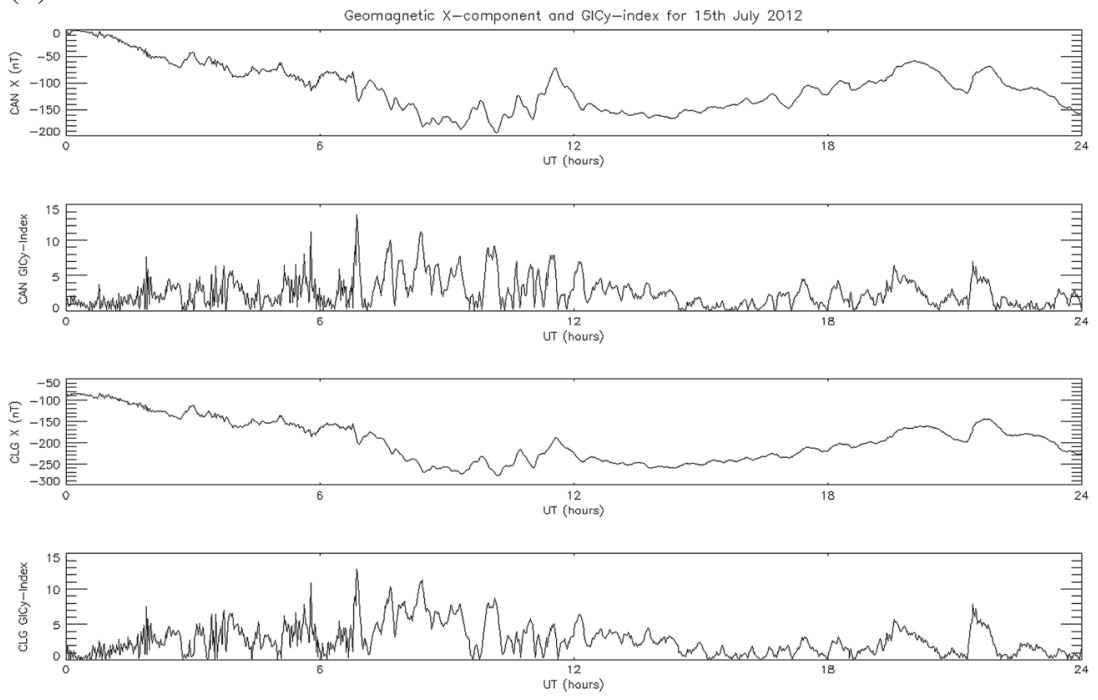

Figure 3. Geomagnetic field $x$-component and associated $\mathrm{GIC}_{y}$ indices for Canberra and Culgoora, respectively, for (a) 14 July and (b) 15 July 2012 (UT). The Canberra magnetometer data were provided courtesy of Geoscience Australia.

fields of $\sim 0.06-0.07$ volts per kilometer. Marshall et al. [2011] presented results of GIC indices observed from a number of Australian region magnetometer locations, finding $\mathrm{GIC}_{y}$ indices 6-10 times this value during significant space weather storms of the past two solar cycles. This would suggest currents of 25 Amps minimum may be observed at these monitoring locations during significant geomagnetic storms and possibly larger during extreme storms. Furthermore, Pulkkinen et al. [2012] presented geoelectric field simulations of extreme storm scenarios for both high and low conductive grounds and super- and sub-auroral electrojet locations giving values ranging from approximately 0.5 to 15 volts per kilometer across the four different scenarios. These values are about
8-200 times as large as the geoelectric fields of $\sim 0.06-$ 0.07 volts per kilometer associated with the currents of 4-5 amperes shown in Figures $4 a$ and $5 a$. Hence, currents of 30 amperes minimum are likely at the monitoring locations presented in this study during extreme storms and possibly considerably larger depending on the extent of expansion of the auroral oval.

[18] Masoum and Moses [2008] presented an analysis of a laboratory transformer under exposure to simulated GIC conditions concluding that transformer losses rise sharply even under relatively small GIC input. Thomson et al. [2005] reported that postevent analysis of GICs observed in the Scottish power network during the 2003 Halloween storm suggested that currents of 25 amperes now constitute a 
(a)

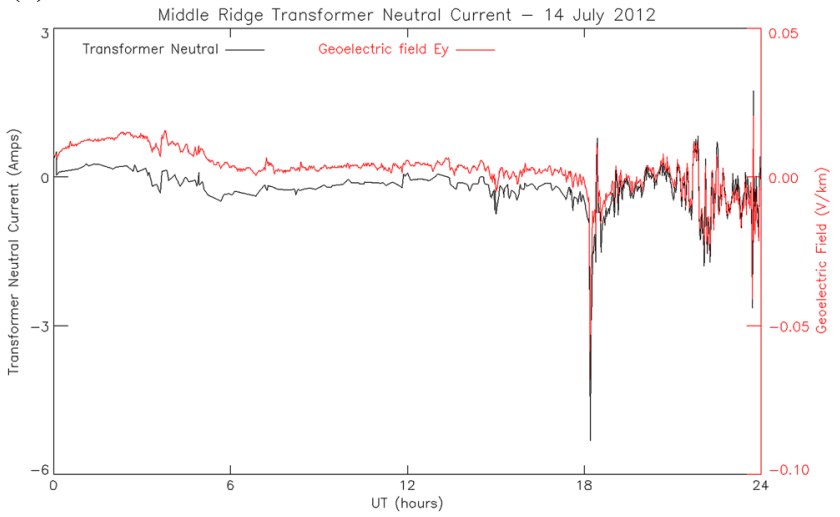

(b)

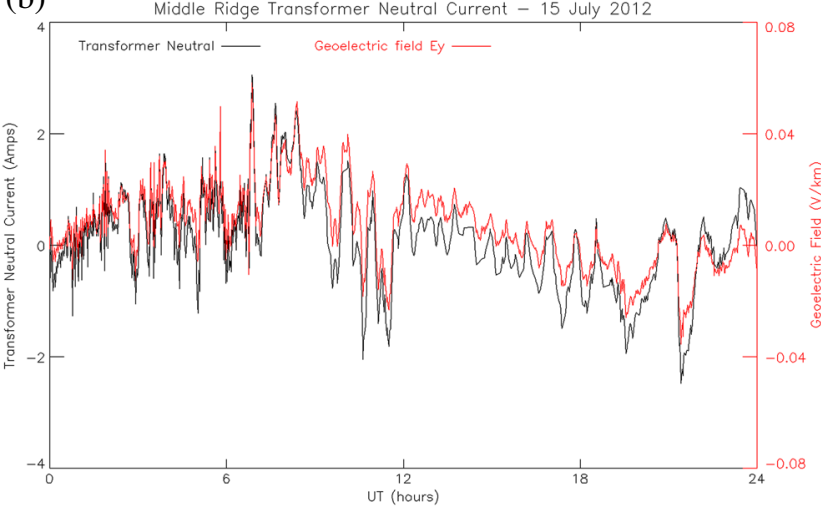

Figure 4. Transformer neutral current from Powerlink's substation at Middle Ridge, Queensland plotted with the geoelectric field, $E_{y}$, in red derived from Culgoora magnetometer data for the modest geomagnetic storm of (a) 14 July and (b) 15 July 2012 (LT).

"concern threshold" for this network. Marshall et al. [2012] presented results of GICs measured in the New Zealand network showing faults occurring in association with GICs of up to 20 amperes flowing in parts of the network. There is no one threshold applicable to all systems due to factors such as system load and transformer design; however, these studies would suggest that GICs with magnitudes of tens of amperes warrant further consideration.

[19] The amplitude of the current in Figures $4 \mathrm{a}$ and $5 \mathrm{a}$ associated with the SI event was slightly higher for the monitor in QLD than the higher latitude monitor in SA. The SI event is the ground signature of enhanced magnetopause currents, which are typically more global in nature and have less variation with latitude than the current systems flowing during the main storm phase [Kappenman, 2003; Marshall et al., 2012]. Hence, comparable currents such as those observed for the SI event at both monitoring sites is understandable. The majority of the current maxima occurring during the storm main phase and period of sustained southward IMF $B_{z}$ are typically larger at the higher latitude site of Figure 5 as might be expected. The relative amplitude of the currents between monitoring locations is dependent on many factors and more comprehensive modeling is required to understand their distribution within the Australian network.

[20] The similarity between the geoelectric field derived from magnetometer stations some distance away from the in situ GIC monitors suggests that the assumed plane wave model and uniform conductivity is reasonable in the situations presented in this study. However, the geoelectric field shown in Figure 4a exhibits a long period (nearly diurnal) trend that appears to be overestimated with respect to the measured GIC. One possible explanation is that the conductivity value used is lower than should be used for such long period signals with relatively large skin-depths. Improved conductivity models such as those in Viljanen and Pirjola [1994], Thomson et al. [2005], and Ngwira et al. [2008] are likely to improve this discrepancy. In addition, the results presented in Figures 5 and 6 would suggest that the uniform geoelectric field assumption is less applicable over large distances such as those
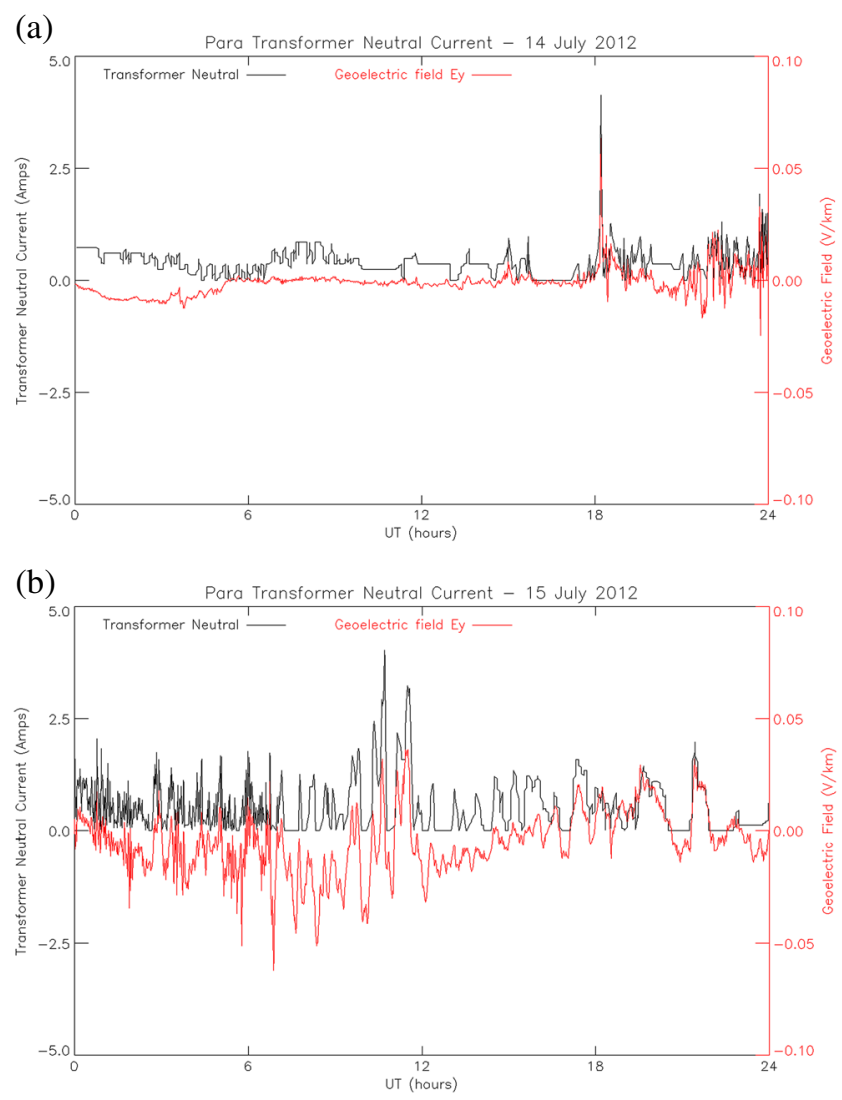

Figure 5. Transformer neutral current from Electranet's substation at Para, South Australia plotted with the geoelectric field, $E_{y}$, in red derived from Canberra magnetometer data for the modest geomagnetic storm of (a) 14 July and (b) 15 July 2012 (UT). Only positive currents were being recorded by the in situ GIC monitor during this event. 


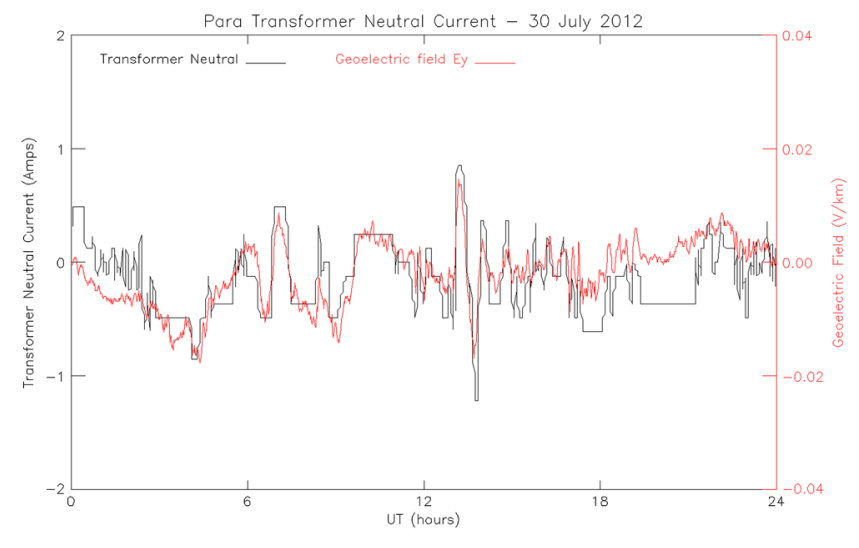

Figure 6. Transformer neutral current from Electranet's substation at Para, South Australia plotted with the geoelectric field, $E_{y}$, in red derived from Canberra magnetometer data for 30 July 2012 (UT). Both positive and negative currents were being recorded by the in situ GIC monitor.

between Canberra and Para. Furthermore, during geomagnetic storm scenarios, the auroral oval expands and auroral electroject currents move further equatorward. Under intense geomagnetic activity, the incident plane wave model may be less applicable. Modeling more appropriate ionospheric current geometry such as demonstrated by Pirjola [1982, 2002], Pirjola and Viljanen [1998], and Viljanen et al. [1999] would provide better estimates of the geoelectric field under these circumstances.

[21] To our knowledge, no reported fault within the Australian power network has been directly attributed to space weather. Prior to the failure of a transformer in New Zealand in 2001, network faults had not been attributed to space weather [Béland and Small, 2004]. Postevent analysis identified space weather as part of the cause for the faults that occurred during the geomagnetic storm of 6 November 2001 [Béland and Small, 2004; Marshall et al., 2012]. Anecdotal evidence that space weather may have directly impacted power networks within Australia are contained in reports from a manager within a southern QLD DNSP Distribution Network Service Provider recalling strange failures of super-long ( 300 kilometers) low resistive 66 kilovolt lines in southern QLD from Roma to St. George in the late 1980s when there were evidences of bright auroras above Dalby $\left(\sim 27^{\circ} \mathrm{S}\right)$ from geomagnetic storms at the time (personal communication, G. Ivanovich, Ergon Energy, QLD). These failures happened several times during morning hours and have never been understood. These events would appear consistent with large GICs occurring during intense storms such as those of March, 1989. It would appear possible that space weather may have contributed to faults within the Australian network but have been reported as "cause unknown" or attributed to another phenomena.
[22] The results presented in this paper show that GICs produced by space weather are readily observable in parts of the Australian power grid, having amplitudes sufficient to be of concern during extreme geomagnetic storm activity. Further monitoring and more complex modeling is required to better understand this phenomenon and determine the most vulnerable parts of the Australian power network. AEMO coordinates the National Energy Market shown in Figure 1a, which does not include the large power network of Western Australia that is owned and operated by Western Power. An independent study conducted by Western Power [Nga and Pearcy, 2012] advises further investigation into GICs within Western Power's network and the installation of in situ monitors. Furthermore, consideration should be given to appropriate management strategies for significant space weather activity. The BOM's space weather branch and AEMO are working toward such procedures; however, a better understanding is required to optimize a response.

\section{Conclusion}

[23] This paper provides results from some of the first in situ GIC monitors installed at strategic locations within the Australian power network as part of an initiative to better understand their impact in this region. Transformer neutral currents from these monitors are compared with the geoelectric field derived from the closest available geomagnetic observatory during a modest geomagnetic storm, showing the first observational evidence of space weather well correlated with GICs in the Australian power network. The transformer neutral currents show a high degree of similarity with the geoelectric field during this storm, having correlation coefficients as high as 0.93 . Current maxima of 4-5 amperes were observed in association with geoelectric field values of $\sim 0.06-0.07$ volts per kilometer. The maximum current that occurred during the storm was in association with a geomagnetic sudden impulse event. Currents of tens of amperes are anticipated at these monitoring locations during more severe geomagnetic activity.

[24] Acknowledgments. The authors would like to thank the Australian Energy Market Operator and members of the Power System Security Working Group for supporting this study. The authors would like to acknowledge the contributions of Malcolm Van Harte of Eskom, South Africa and Grujica Ivanovich of Ergon Energy, Queensland. The authors would also like to thank the Geomagnetism group of Geoscience Australia for provision of Canberra magnetometer data used in this study. The authors would also like to thank the World Data Centre (WDC) for Geomagnetism, Kyoto, Japan, and NOAA's Space Weather Prediction Centre, United States, for provision of geomagnetic indices used in this study. The authors would like to acknowledge the ACE satellite group for solar wind data used in this study.

\section{References}

Beamish, D., T. D. G. Clark, E. Clark, and A. W. P. Thomson (2002), Geomagnetically induced currents in the UK: Geomagnetic variations and surface electric fields, J. Atmos. Sol. Terr. Phys., 64, 1779-1792, doi:10.1016/S1364-6826(02)00127-X. 


\section{MARSHALL ET AL.: GICS IN AUSTRALIAN POWER NETWORK}

Béland, J., and K. Small (2004), Space weather effects on power transmission systems: The cases of Hydro-Quebec and Transpower New Zealand Ltd., in Effects of Space Weather on Technology Infrastructure, edited by I. A. Eaglis, pp. 287-299, Kluwer Acad., Dordrecht, Netherlands.

Bolduc, L. (2002), GIC observations and studies in the Hydro-Québec power system, J. Atmos. Sol. Terr. Phys., 64, 1793-1802, doi:10.1016/ S1364-6826(02)00128-1.

Boteler, D. H., R. J. Pirjola, and H. Nevanlinna (1998), The effects of geomagnetic disturbances on electrical systems at the Earth's surface, Adv. Space Res., 22, 17-27, doi:10.1016/S0273-1177(97)01096-X.

Cagniard, L. (1953), Basic theory of the magneto-telluric method of geophysical prospecting, Geophysics, 18(3), 605-635, doi:10.1190/ 1.1437915 .

Campbell, W. H., C. E. Barton, F. H. Chamalaun, and W. Welsh (1998), Quiet-day ionospheric currents and their application to upper mantle conductivity in Australia, Earth Planets Space, 50, 347-360.

Dalzell, M. (2011), DC currents in the New Zealand AC power system, paper presented at Conference and Exhibition, Electr. Eng. Assoc., Auckland, N. Z., 23-24 June.

Elovaara, J., A. Viljanen, T. Makinen, S. Larsson, P. Lindblad, R. Pirjola, and B. Kielen (1992), Geomagnetically induced currents in the Nordic power system and their effects on equipment, control, protection and operation, paper presented at CIGRE Conference, Paris.

Gaunt, C. T., and G. Coetzee (2007), Transformer failures in regions incorrectly considered to have low GIC risk, in Power Tech, 2007 IEEE Lausanne, pp. 807-812, doi:0.1109/PCT.2007.4538419.

Gorniak, H. (2011), Update on assessment of potential impact of coronal mass ejection, solar flares, geomagnetic storms on the NEM power system, AEMO Communication No. 914, December 2011, Australian Energy Market Operator Limited, Melbourne, Australia.

Hejda, P., and J. Bochnicek (2005), Geomagnetically induced pipe-tosoil voltages in the Czech oil pipelines during October-November 2003, Ann. Geophys., 23, 3089-3093.

Kappenman, J. G. (2003), Storm sudden commencement events and the associated geomagnetically induced current risks to groundbased systems at low-latitude and midlatitude locations, Space Weather, 1(3), 1016, doi:10.1029/2003SW000009.

Kappenman, J. G. (2004), Space weather and the vulnerability of electric power grids, in Effects of Space Weather on Technology Infrastructure, edited by I. A. Eaglis, pp. 257-286, Kluwer Acad., Dordrecht, Netherlands.

Kappenman, J. G. (2005), An overview of the impulsive geomagnetic field disturbances and power grid impacts associated with the violent Sun-Earth connection events of 29-31October 2003 and a comparative evaluation with other contemporary storms, Space Weather, 3, S08C01, doi:10.1029/2004SW000128.

Lam, H. L., D. H. Boteler, and L. Trichtchenko (2002), Case studies of space weather events from their launching on the Sun to their impacts on power systems on the Earth, Ann. Geophys., 20, 1073-1079, doi:10.5194/angeo-20-1073-2002.

Lehtinen, M., and R. Pirjola (1985), Currents produced in earthed conductor networks by geomagnetically-induced electric fields, Ann. Geophys., 3, 479-484.

Liu, C.-M., L. Liu, and R. Pirjola (2009a), Geomagnetically induced currents in the high voltage power grid in China, IEEE Trans. Power Delivery, 24(4), 2368-2374, doi:10.1109/TPWRD.2009.2028490.

Liu, C.-M., L.-G. Liu, R. Pirjola, and Z.-Z. Wang (2009b), Calculation of geomagnetically induced currents in mid- to low-latitude power grids based on the plane wave method: A preliminary case study, Space Weather, 7, S04005, doi:10.1029/2008SW000439.

Marshall, R. A., C. L. Waters, and M. D. Sciffer (2010), Spectral analysis of pipe-to-soil potentials with variations of the Earth's magnetic field in the Australian region, Space Weather, 8, S05002, doi:10.1029/ 2009 SW000553.

Marshall, R. A., E. A. Smith, M. J. Francis, C. L. Waters, and M. D. Sciffer (2011), A preliminary risk assessment of the Australian region power network to space weather, Space Weather, 9, S10004, doi:10.1029/ 2011SW000685.

Marshall, R. A., M. Dalzell, C. L. Waters, P. Goldthorpe, and E. A. Smith (2012), Geomagnetically induced currents in the New Zealand power network, Space Weather, 10, S08003, doi:10.1029/2012SW000806.

Martin, B. A. (1993), Mitigation of telluric effects on a buried pipeline, Corrosion, 49(4), 343-350, doi:10.5006/1.3316059.
Masoum, M. A. S., and P. S. Moses (2008), Influence of Geomagnetically Induced Currents on Three-Phase Power Transformers, AUPEC0 conference proceedings, paper P-242, 14-17 December 2008, University of New South Wales, Sydney, Australia.

Nga, J., and T. Pearcy (2012), Geomagnetic Induced Current Preliminary Study, Rep., DM: 9051805, Western Power, Perth, Western Australia.

Ngwira, C. M., A. Pulkkinen, L.-A. McKinnell, and P. J. Cilliers (2008), Improved modeling of geomagnetically induced currents in the South African power network, Space Weather, 6, S11004, doi:10.1029/ 2008SW000408.

Ngwira, C. M., L.-A. McKinnell, P. J. Cilliers, A. Viljanen, and R. Pirjola (2009), Limitations of the modeling of geomagnetically induced currents in the South African power network, Space Weather, 7, S10002, doi:10.1029/2009SW000478.

Pirjola, R. (1982), Electromagnetic induction in the Earth by a plane wave or by fields or line currents harmonic in time and space, Geophysica, 18, 1-161.

Pirjola, R., and A. Viljanen (1998), Complex image method for calculating electric and magnetic fields produced by an auroral electrojet of finite length, Ann. Geophys., 16, 1434-1444.

Pirjola, R. (2000), Geomagnetically induced currents during magnetic storms, IEEE Trans. Plasma Sci., 28(6), 1867-1873, doi:10.1109/27.902215

Pirjola, R. (2002), Review on the calculation of surface electric and magnetic fields and of geomagnetically induced currents in ground-based technological systems, Surv. Geophys., 23, 71-90, doi:10.1023/A:1014816009303.

Pirjola, R. (2005), Effects of space weather on high-latitude ground systems, Adv. Space Res., 36, 2231-2240, doi:10.1016/j.asr.2003.04.074.

Pulkkinen, A., A. Thomson, E. Clarke, and A. McKay (2003), April 2000 geomagnetic storm: Ionospheric drivers of large geomagnetically induced currents, Ann. Geophys., 21, 709-717, doi:10.5194/ angeo-21-709-2003.

Pulkkinen, A., S. Lindahl, A. Viljanen, and R. Pirjola (2005), Geomagnetic storm of 29-31 October 2003: Geomagnetically induced currents and their relation to problems in the Swedish high-voltage power transmission system, Space Weather, 3, S08C03, doi:10.1029/ 2004 SW000123.

Pulkkinen, A. A., E. Bernabeu, J. Eichner, C. Beggan, and A. W. P. Thomson (2012), Generation of 100-year geomagnetically induced current scenarios, Space Weather, doi:10.1029/2011SW000750, in press.

Thomson, A. W. P., R. McKay, E. Clarke, and S. J. Reay (2005), Surface electric fields and geomagnetically induced currents in the Scottish Power grid during the 30 October 2003 geomagnetic storm, Space Weather, 3, S11002, doi:10.1029/2005SW000156.

Torta, J. M., L. Serrano, J. R. Regué, A. M. Sánchez, and E. Roldán (2012), Geomagnetically induced currents in a power grid of northeastern Spain, Space Weather, 10, S06002, doi:10.1029/ 2012SW000793.

Trichtchenko, L., and D. H. Boteler (2004), Modeling geomagnetically induced currents using geomagnetic indices and data, IEEE Trans. Plasma Sci., 32(4), 1459-1467, doi:10.1109/TPS.2004.830993.

Trichtchenko, L. (2005), November 2004 Space Weather Events: RealTime Observations And Forecasts, in proceedings ESWW-II meeting, 14 - 18th November 2005, ESA-ESTEC, The Netherlands.

Trivedi, N. B., et al. (2007), Geomagnetically induced currents in an electric power transmission system at low latitudes in Brazil: A case study, Space Weather, 5, S04004, doi:10.1029/2006SW000282.

Viljanen, A., and R. Pirjola (1994), On the possibility of performing studies on the geoelectric and ionospheric currents using induction in power systems, J. Atmos. Terr. Phys., 56, 1483-1491.

Viljanen, A., O. Amm, and R. Pirjola (1999), Modeling geomagnetically induced currents during different ionospheric situations, J. Geophys. Res., 104, 28,059-28,071, doi:10.1029/1999JA900337.

Wang, L. J., and F. H. Chamalaun (1995), A magnetotelluric traverse across the Adelaide geosyncline, Exploration Geophys., 26, 539-546.

Wang, L.J., and F. E. M. Lilley (1999), Inversion of magnetometer array data by thin-sheet modelling, Geophys. J. Int., 137, 128-138.

Watari, S., et al. (2009), Measurements of geomagnetically induced current in a power grid in Hokkaido, Japan, Space Weather, 7, S03002, doi:10.1029/2008SW000417.

Waters, C. L., R. A. Marshall, and R Hoogsteyns. (2006), Telluric currents induced in a North Queensland gas pipeline by geomagnetic variations, paper presented at WARS2006, Natl. Comm. for Radio Sci., Leura, N. S. W., Australia, 15-17 Feb. 


\section{MARSHALL ET AL.: GICS IN AUSTRALIAN POWER NETWORK}

Wik, M., A. Viljanen, R. Pirjola, A. Pulkkinen, P. Wintoft, and H. Lundstedt (2008), Calculation of geomagnetically induced currents in the $400 \mathrm{kV}$ power grid in southern Sweden, Space Weather, 6, S07005, doi:10.1029/2007SW000343.
Zanussi, P. (1993), Effects of Geomagnetically Induced Current on North Queensland Transmission Lines, M. Eng. Sc. thesis, Dept. of Electr. and Comp. Eng., James Cook University, North Queensland, Australia. 